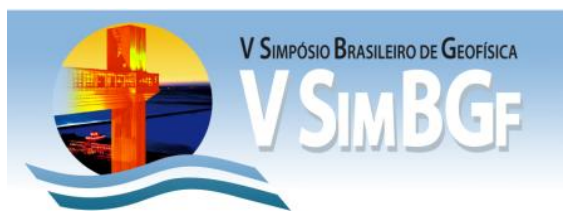

\title{
Textura e distribuição tamanho de poro incorporadas na modelagem de eletrofácies objetivando uma melhor estimativa da permeabilidade em reservatórios carbonáticos
}

\author{
Marcelo Torrez ${ }^{1}$; Eduardo Barreto ${ }^{1}$ Abel Carrasquilla ${ }^{2} \&$ Pablo Sanchez $^{3}$ \\ ${ }^{1}$ Petrobras -CENPES, ${ }^{2}$ UENF - Macaé, ${ }^{3}$ Paradigm - Brasil
}

Copyright 2012, SBGf - Sociedade Brasileira de Geofísica

Este texto foi preparado para a apresentação no V Simpósio Brasileiro de Geofísica, Salvador, 27 a 29 de novembro de 2012. Seu conteúdo foi revisado pelo Comitê Técnico do V SimBGf, mas não necessariamente representa a opinião da SBGf ou de seus associados. É proibida a reprodução total ou parcial deste material para propósitos comerciais sem prévia autorização da SBGf.

\section{Resumo}

The continuous improvement in the acquisition and processing of Resistivity Image (RI) and Nuclear Magnetic Resonance (NMR) logs allows the development of approaches that enable relevant information from these tools, which can be used in the core-log correlation studies to enrich the characterization of carbonate reservoirs. Thus, the objectives of this study were to establish a workflow that allows the incorporation of information, such as texture derived from the processing of RI log and pore size distribution from the NMR log, into the rock-typing classification, using clustering algorithms and statistical techniques. All the derived models were calibrated with basic laboratory petrophysical core analyses. On the other hand, the depositional model of the studied area corresponds to a carbonate platform of Campos Basin, represented by high-energy benches with shoalling upward cycles, where the predominant facies varies from peloidal packstones to oolitic grainstones and cemented grainstones. In a dataset with three boreholes, the reference well $A$ was divided in three rock types, based on this, it was performed a clustering analysis on $\mathrm{RI}$ log aiming to identify textural facies. In this form, the obtained results provide essential information in the characterization of this carbonate reservoir, which aim to diminish the uncertainties of the estimation of the hydrocarbons in place and production.

\section{Introdução}

Os constantes avanços na área de aquisição e processamento de perfis RI e NMR geram uma demanda para o desenvolvimento de fluxos de trabalho que permitam incorporar informações relevantes destas ferramentas nos estudos de correlação rocha-perfil, promovendo, desta forma, melhorias na caracterização de reservatórios.

Os perfis RI têm sido utilizados para estudos geológicos por várias décadas. Durante todo este período, a identificação de fácies sedimentológicas e/ou estruturais estava limitada somente a uma análise visual do interprete. Nestes últimos anos, esta técnica foi progredindo paulatinamente até incorporar rotinas automáticas que permitiram capturar com alta resolução e, de forma mensurável, características sedimentológicas e estruturais da rocha. Por outro lado, as leituras do perfil NMR independem da densidade mineral e permitem caracterizar o tipo de fluido e porosidade da matriz da rocha. A física de medição desta ferramenta é baseada na polarização dos átomos de hidrogênio, onde a constante do tempo da razão de decaimento de energia (relaxação transversal, $T_{2}$ ) é função da distribuição do tamanho de poros e não da litologia. A curva de distribuição do tempo de relaxação $T_{2}$ permite inferir vários parâmetros dos reservatórios, entre eles a porosidade, permeabilidade, etc. Em conclusão, características sedimentológicas e petrofísicas das rochas, tais como textura e distribuição de tamanho de poro, estimadas nos perfis RI e NMR, respectivamente, fornecem informações valiosas para caracterizar 0 reservatório, podendo ser incorporadas nos trabalhos de correlação rocha-perfil via inteligência artificial ou técnicas estatísticas.

Este estudo foi realizado utilizando dados de laboratório e perfis geofísicos de poço de um reservatório presente em rochas depositadas num ambiente de plataforma carbonática extensa, com mais de $1500 \mathrm{~km}$ de extensão ao longo da costa das Bacias de Campos e Santos (Figura 1). A evolução sedimentar desta plataforma foi condicionada por estruturas da seção pré-albiana (Baixo de São Tomé, Altos Internos e Externos, lineamentos NW e NE). A movimentação dos evaporitos foi influenciada pela carga sedimentar, inclinação do substrato e reativações de falhamentos (direção NW/SE), controlando a geometria e distribuição de fácies.

Assim, o presente trabalho tem como objetivo principal estabelecer um fluxograma que permita incorporar os perfis RI e NMR nas rotinas de correlação rocha-perfil. Desta forma, pretende quantificar o grau heterogeneidade do reservatório em termos de variação textural nas imagens de poço e distribuição de tamanho de poro no perfil de NMR, para a construção de modelos de eletrofácies que permitam refinar a permeabilidade em reservatórios carbonáticos.

\section{Metodologia}

A metodologia aplicada neste trabalho pode ser simplificada em dois passos:

1) processamento de diferentes grupos de dados para estimar modelos de eletrofácies supervisionados pela descrição de litofacies de testemunho. Desta forma, pretende-se incorporar a classificação automática de texturas e a distribuição de tamanho para que, posteriormente, estes modelos sejam treinados em intervalos com testemunho e extrapolados com técnicas de agrupamento com perfis convencionais (Figura 2);

2) posteriormente serão construídos modelos de permeabilidade supervisionados pelos resultados de eletrofácies e petrofísica básica de laboratório.

A identificação de texturas no perfil RI utiliza uma abordagem estatística, aplicando a metodologia proposta por Gagalowicz (1987), a qual facilita a extração de 
texturas em função dos parâmetros estatísticos de histogramas e autocovariância. Os histogramas permitem quantificar o contraste das texturas e a função de autocovariância fornece informação sobre a orientação e tamanho das texturas primitivas. Este método permite criar imagens sintéticas para posterior comparação com as imagens originais, com o objetivo de controlar a representatividade dos parâmetros de textura utilizados. Estes parâmetros estatísticos podem caracterizar quase todas as possíveis texturas, mesmo que elas não tenham sido observadas anteriormente (Ye \& Rabiller, 2001)

A distribuição do tamanho de poro foi estimada com o perfil NMR, onde foi gerado um histograma dos canais do $\mathrm{T}_{2 \text { dist }}$ com uma janela de procura de cumprimento igual a resolução vertical deste perfil. Posteriormente, o histograma foi agrupado com o algoritmo MRGC (Multi Resolution Graph - Based Clustering) e calibrado com dados de porosidade de testemunho para obter um modelo de eletrofácies que reproduza a variação de tamanho de poro em função da porosidade.

As ferramentas estatísticas usadas para fazer o tratamento, exploração e extrapolação dos resultados são o método de agrupamento MRGC e a técnica KNN (Kernel Nearest Neighbour) proposta por Ye et al. (1998). Este tipo de técnicas estatísticas permitem propagar informações geológicas e petrofísicas para poços com similares formações geológicas (Ye \& Rabiller, 2000).

Finalmente, os modelos de eletrofácies que integram a classificação de textura e distribuição de tamanho de poro, e petrofisica básica de testemunho foram usados como variáveis de entrada para predição de permeabilidade do reservatório.

\section{Resultados}

A análise de textura de imagem é uma classificação quantitativa da textura elétrica da formação automática, obtida a partir de perfis RI (Ye et al., 1998). Numa primeira etapa, o algoritmo aprende todas as texturas e um modelo sintético é gerado. Essas texturas são classificadas para criar um mapa textural ou Tmap (100 classes mais representativas). Posteriormente, um perfil de textura pode ser criado, atribuindo uma classe textural para cada intervalo em profundidade, onde para cada intervalo é atribuído uma coluna e valor. A fim de facilitar a utilização baseada em seus aspectos texturais, o Tmap foi dividido em 8 grupos, de acordo as suas semelhanças visuais e desta forma poder ser comparado com as litofácies de testemunho

Após a normalização da imagem, o passo seguinte é a extração dos parâmetros estatísticos de histograma e autocovariância. Estes parâmetros são estimados para todos os patins da imagem de uma mesma camada, na qual a janela de controle é definida de acordo com a orientação local do patim da imagem. O processamento depende de duas variáveis que são o tamanho e incremento da janela de controle. Neste trabalho em especifico, esta janela foi ajustada para que a variação textural do perfil de imagem possa ser comparada com a curva de litofácies de testemunho. Para efeitos de visualização, estes parâmetros são apresentados como uma curva de distribuição (Feature log).

Os primeiros 8 elementos correspondem ao histograma que descreve a distribuição de cores (8 níveis de cor cinza) e o restante dos elementos representa a matriz de autocovariância que descreve o arranjo espacial dos pixels da imagem. Baseados na forma e aparência do feature log, foi realizada uma análise descritiva, onde pode se observar que as texturas homogêneas têm uma aparência onde predomina a alta freqüência da curva, quanto que o comportamento das texturas heterogêneas é inverso, ou seja, a curva apresenta uma baixa frequência marcada pela sinuosidade da curva (Figura 3). As texturas sintéticas foram classificadas pelo método de agrupamento MRGC, criando um mapa textural ou Tmap, que foi dividido em 8 grupos. O perfil de textura pode ser criado atribuindo classes texturais para cada intervalo de profundidade, onde cada uma é associada a uma profundidade. Desta forma, se obtem um perfil contínuo que denota a variabilidade do reservatório em termos de textura. Posteriormente, os grupos de texturas foram comparados com as litofácies de testemunho, facilitando o entendimento do significado das mesmas em termos litológicos. Da mesma forma, a análise realizada sobrepondo graficamente a permeabilidade de laboratório e a classificação de texturas, permitiu estabelecer unidades de fluxo (FU) no reservatório, concordantes com as variações de textura interpretadas nos perfis de imagem (Figura 4).

Um modelo foi testado e agrupado com o método MRGC no poço A para extrapolar as fácies de textura para o poço B. Este modelo encontra-se supervisionado pela classificação de texturas e treinado com perfis convencionais (GR, NPHI, RHOZ, DTCO e PEF). Esta modelagem foi realizada em duas etapas: primeiramente, 25 clusters foram estimados e, a seguir, agrupados em 7 novos clusters através de cross plots entre os perfis de $\mathrm{RHOZ}$ e NPHI, que permitiram representar as classes texturais interpretadas no perfil RI. Assim, a Figura 5 mostra os resultados da extrapolação das texturas do poço A para o poço B. As texturas de cor azul claro referem-se a níveis cimentados que encontram-se bem caracterizadas por eventos resistivos conforme o perfil de imagem do poço B. A textura de cor amarela não foi identificada no poço $B$, devido a uma provável descontinuidade lateral de fácies.

A distribuição do tamanho de poro foi estimada com o perfil de NMR, onde foi gerado um histograma dos canais da curva de $\mathrm{T}_{2 \text { dist }}$ (30 bins) com uma janela de processamento de espaçamento igual à resolução vertical do perfil de NMR. O histograma resultante de $\mathrm{T}_{2 \text { dist }}$ foi comparado como os dados de porosidade de perfil e de testemunho respectivamente, onde pode-se observar a presença de ciclos de variação de tamanho de poro no reservatório. Posteriormente, este histograma foi agrupado com a metodologia MRGC e os clusters resultantes foram calibrados com dados de porosidades de testemunho. O resultado desta análise, e uma curva de eletrofácies ( $\left.E_{\text {fac_- }} T_{2 \text { dist }}\right)$, que denota a heterogeneidade do reservatório em função do tamanho do poro. (Figura 6).

O objetivo principal deste trabalho foi o de construir modelos de permeabilidade que integrassem informações texturais $\left(\mathrm{E}_{\mathrm{fac} \_\mathrm{Tex}}\right)$, distribuição de tamanho de poro $\left(E_{\text {fac_ }} T_{2 d i s t}\right)$, petrofisica básica de laboratório e perfis 
convencionais. Este modelo foi construído usando a metodologia de agrupamento de dados MRGC, onde foram usados perfis convencionais(GR, NPHI, RHOZ, DTCO e PEF), supervisionados pela classificação de texturas e dados de permeabilidade de testemunho. A escolha dos perfis de entrada para este modelo esta baseada na observação de cross plots entre a permeabilidade e os perfis convencionais. Inicialmente, foi gerada uma análise de agrupamento com 11 agrupamentos (clusters) o qual foi agrupado em 7 clusters, onde foram identificados 6 classes que correspondem a fácies grainstone (uma delas cimentada), e a outra classe corresponde as fácies packstones. Existe um aprendizado dos dados de permeabilidade e dos valores de perfis convencionais através de MRGC, logo, esta classificação é propagada para o resto da formação. Nesse momento, é possível definir qual Nearest neighbours (KNN) será utilizado (Ye et al., 1998). Ao se comparar os resultados dos diferentes valores de KNN para o mesmo modelo, é possível avaliar a representatividade do modelo (Knecht, 2003) O valor de permeabilidade para cada ponto do perfil é obtido ao se reproduzir o valor de permeabilidade do ponto mais próximo no espaço na medidas de testemunho. A curva de saída foge do padrão apresentado por outros métodos, pelo fato que esta curva não tem suavização e apresenta alguns picos em alguns intervalos (Ye \& Rabiller, 2001). Os 25 clusters gerados objetivam resgatar a heterogeneidade do reservatório em termos de variação de tamanho de poro. A fim de avaliar a representatividade dos resultados e comparar com os dados de permeabilidade de laboratório, foram propostos vários modelos com variação do numero de KNN (Figura 7). Finalmente, o modelo foi extrapolado para o poço $B$, onde pode ser observado que os modelos gerados têm ajustes razoáveis com os dados de permeabilidade das amostras laterais (Figura 8).

\section{Conclusões}

A metodologia empregada neste trabalho de correlação rocha-perfil permitiu incorporar os atributos de texturas dos perfis de imagem elétrica do perfil RI, a distribuição de tamanho de poro do perfil NMR, os perfis convencionais (GR, NPHI, RHOZ, DTCO e PEF) e os dados de testemunho, como subsídio na modelagem de eletrofácies e predição de permeabilidade em reservatórios carbonáticos. Para esta finalidade, foi gerado um modelo sintético de textura definido por o histograma e a função de autocovariância, representados por mapa de textura 1D, o qual foi agrupado e classificado automaticamente com critérios de linearidade, orientação, contraste, tamanho e frequência. Um perfil de eletrofácies de textura foi gerado, o que permitiu registrar a variação da heterogeneidade do reservatório com os perfis de imagem elétrica. $O$ modelo resultante foi comparado com as litofácies de testemunho. A detecção de auto-textura pode ser utilizada diretamente como variável de supervisão na predição de eletrofácies ou propriedades da rocha de (porosidade e permeabilidade). Isto permite predizer a qualidade do reservatório em intervalos não testemunhados ou em poços vizinhos com características geológicas semelhantes. As eletrofácies obtidas da distribuição do perfil NMR, por meio do escalamento da curva de distribuição de T2, promovem um melhor entendimento da relação entre a porosidade, permeabilidade e distribuição de tamanho de poro com as fácies sedimentares. Os modelos gerados por eletrofácies denotaram características de variação tanto na textura quanto na distribuição de tamanho de poro das rochas, permitindo refinar os modelos de permeabilidade e resgatar o grau de heterogeneidade comum nos reservatórios carbonáticos.

\section{Agradecimentos}

À PETROBRAS por ceder os dados de poço de reservatório carbonático da Bacia de Campos e à PARADIGM por la licença acadêmica do software Geolog.

\section{Referências}

Bruhn, C.; Gomes, J.; Lucchese. C. \& Johann, P. 2003. Campos Basin: Reservoir Characterization and Management - Historical Overview and Future Challenges. Offshore Technology Conference, p. 15220, $14 \mathrm{pp}$.

Franco, N. P.; Schuab, F.; Nocchl, G. 2011. Estudo para determinação dos cortes de t2 através da integração entre a distribuição de t2 do perfil de ressonância magnética com a distribuição de garganta de poros do ensaio de pressão capilar por injeção de mercúrio. Relatório do Projeto Análise de dados de reservatórios. Convênio Petrobras/LENEP-UENF/Fundação BioRio, 76 pp.

Gagalowicz A, 1987, Texture modelling applications, The Visual Computers, Vol 3, p. 186-200, Springer Verlag.

Knecht L. 2003, Electrofacies and permeability modeling in carbonate reservoir using image texture analysis and clustering tools, SPWLA, 44 st Annual Logging Symposium, Transaction, paper $\mathrm{H}$.

Ye, S.-J.; Rabiller, P. \& Keskes, N. 1998. Automatic high resolution texture analyze on borehole, SPWLA, 39st Annual Logging Symposium Transaction, paper M.

Ye, S.-J. \& Rabiller, P. 2000. A new tool for eletrofacies analysis: multi-resolution graph-based clustering, SPWLA, 41st Annual Logging Symposium Transaction, paper PP.

Ye, S.-J. \& Rabiller, P. 2001. the interactive use of clustering and modeling to improve permeability prediction, SPWLA, 42st Annual Logging Symposium. Transaction, paper EE.

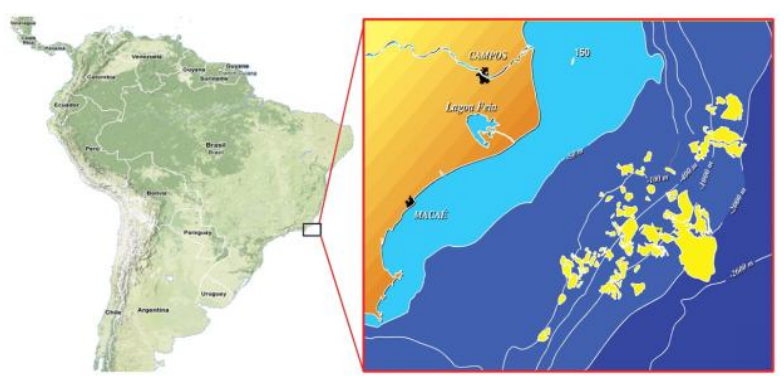

Figura 1. Localização da Bacia de Campos (modificado de Bruhn et al. 2003). 


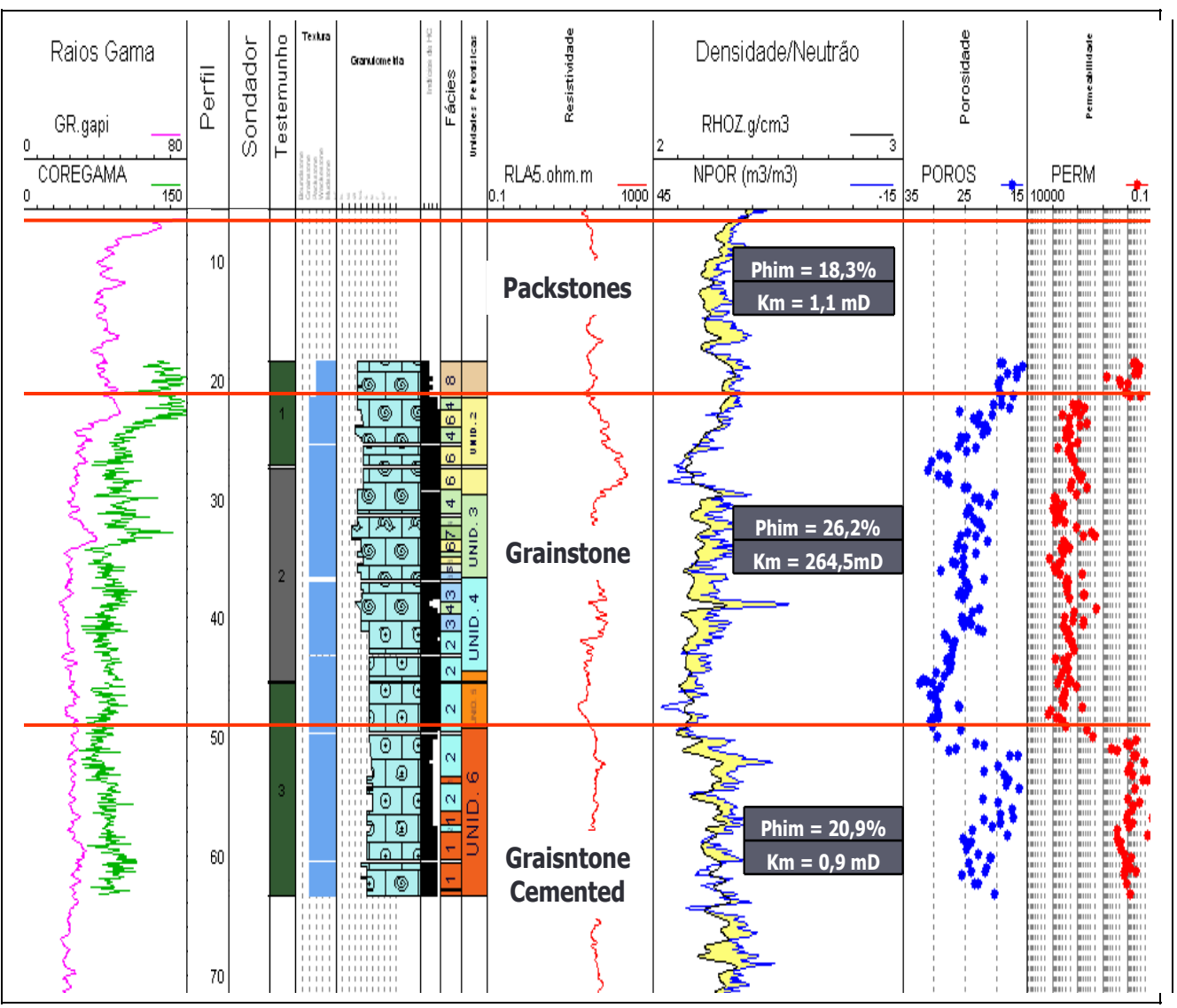

Figura 2. Zoneamento do reservatório e propriedades petrofisicas médias para cada intervalo (modificado de Franco et al.. 2011).

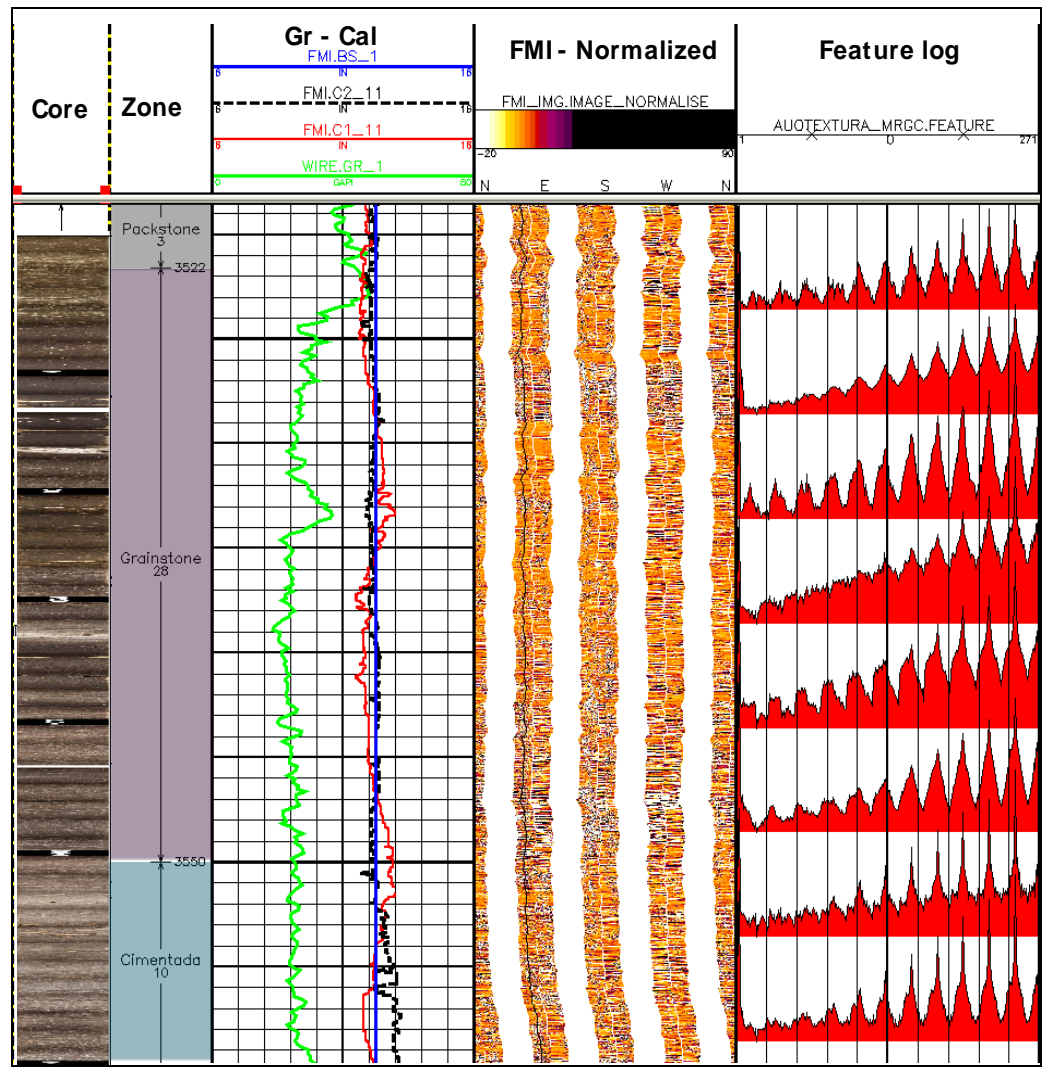

Figura 3. Estimativa dos parâmetros de textura no poço A. 


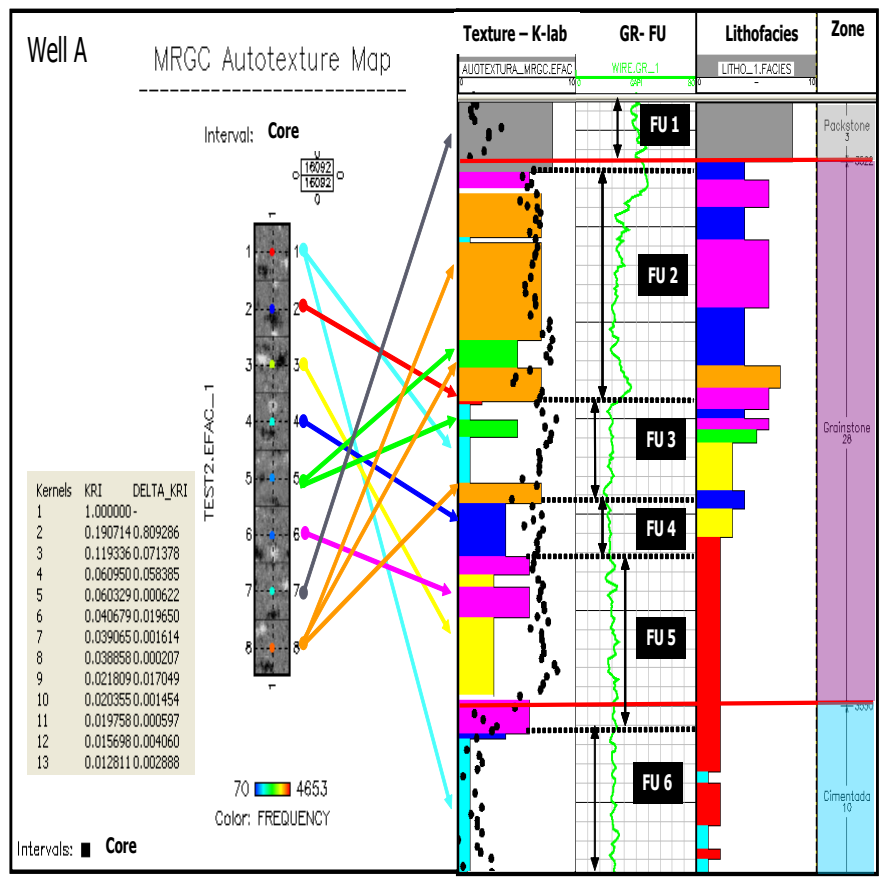

Figura 4. da classificação de texturas com as litofácies de testemunho.

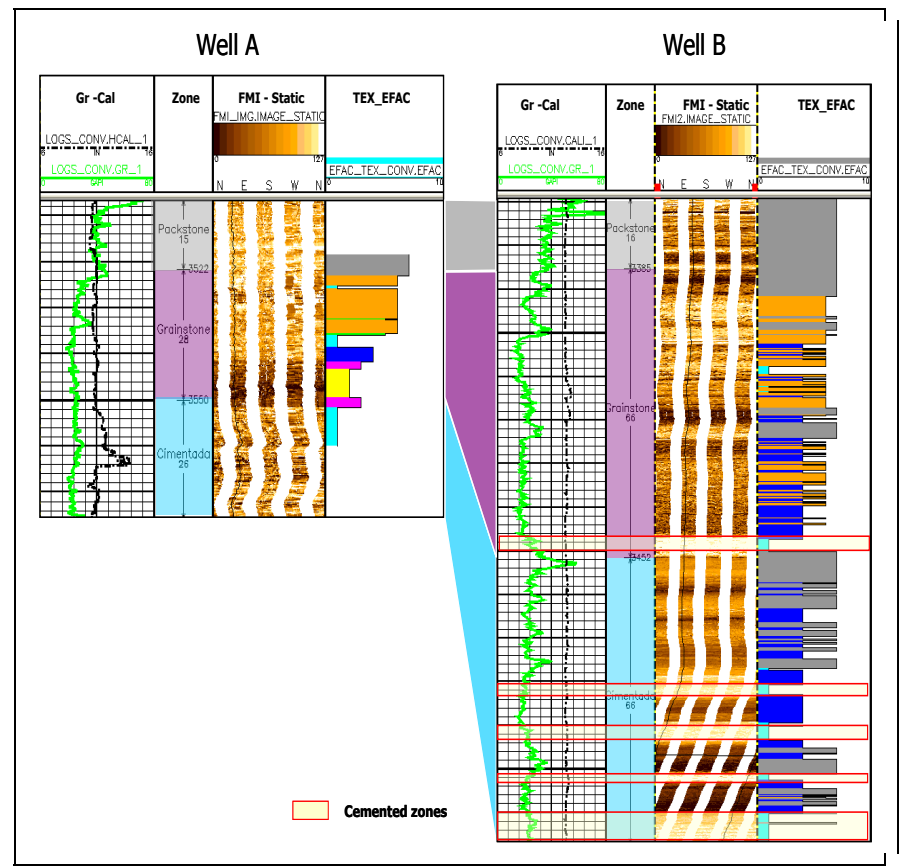

Figura 5. Correlação estratigráfica mostrando a classificação de texturas.

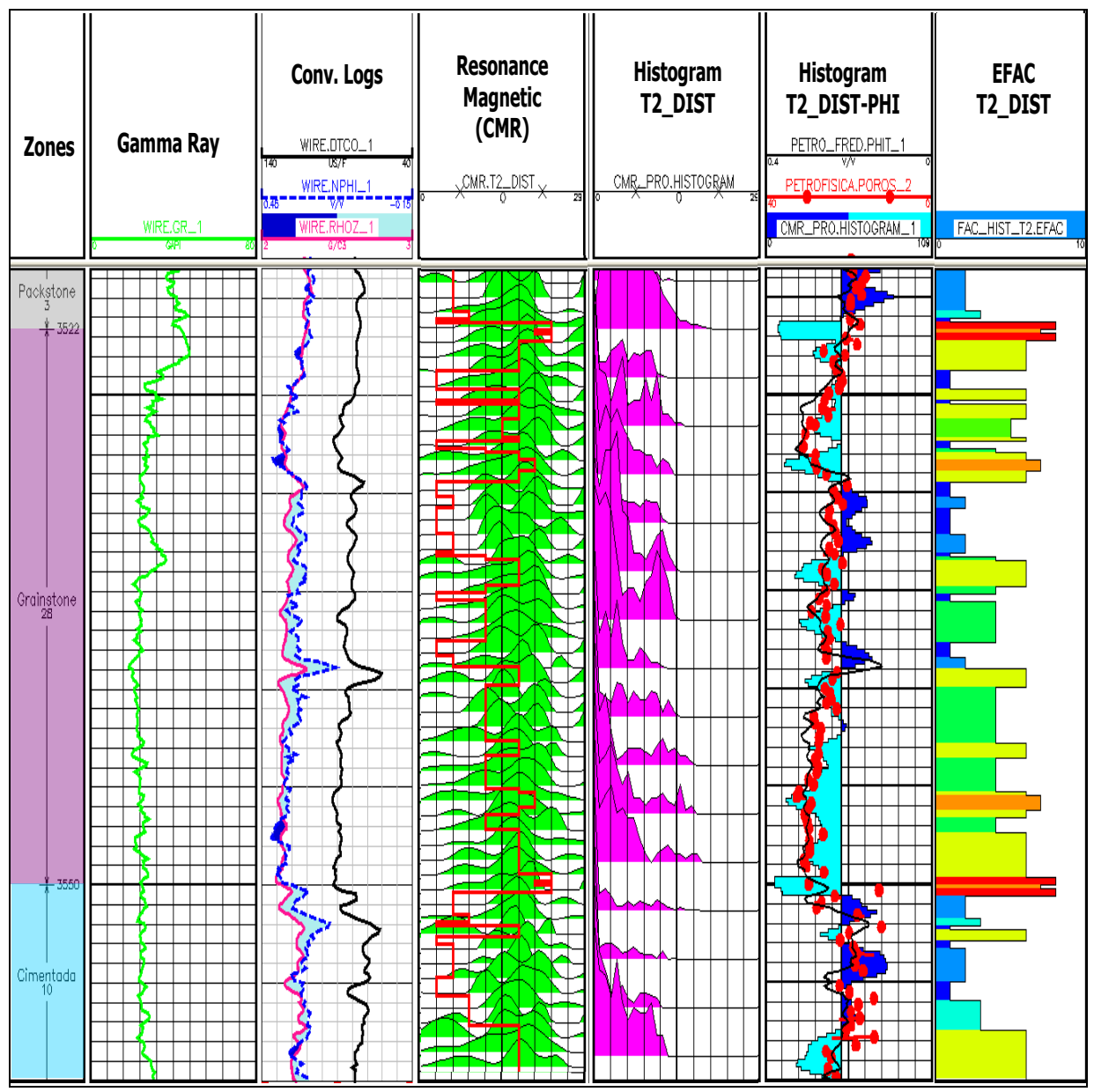

Figura 6. Metodologia para a estimativa da distribuição de tamanho poro com dados de NMR. 


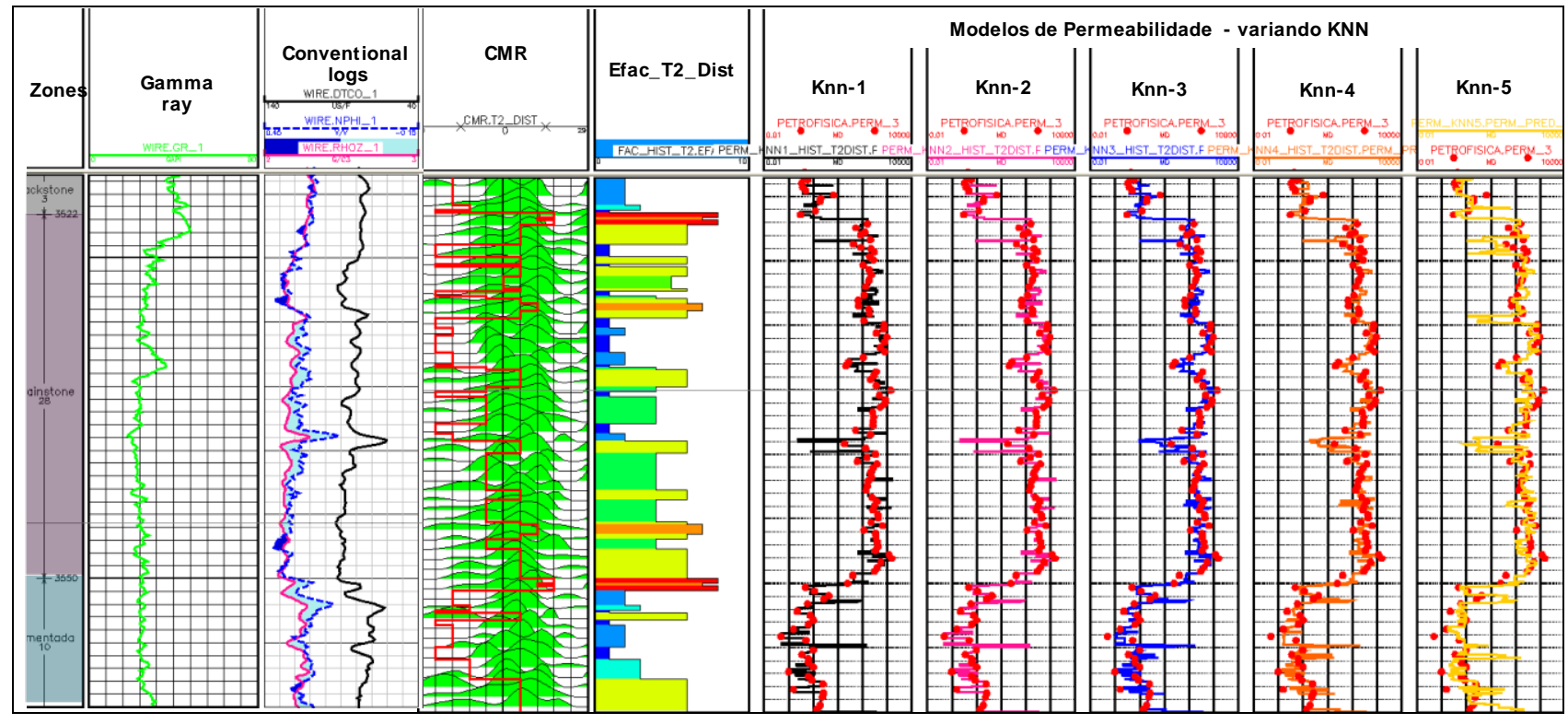

Figura 7. Modelos de permeabilidade variando o KNN (Kernel Nearest Neighbours) no poço A.

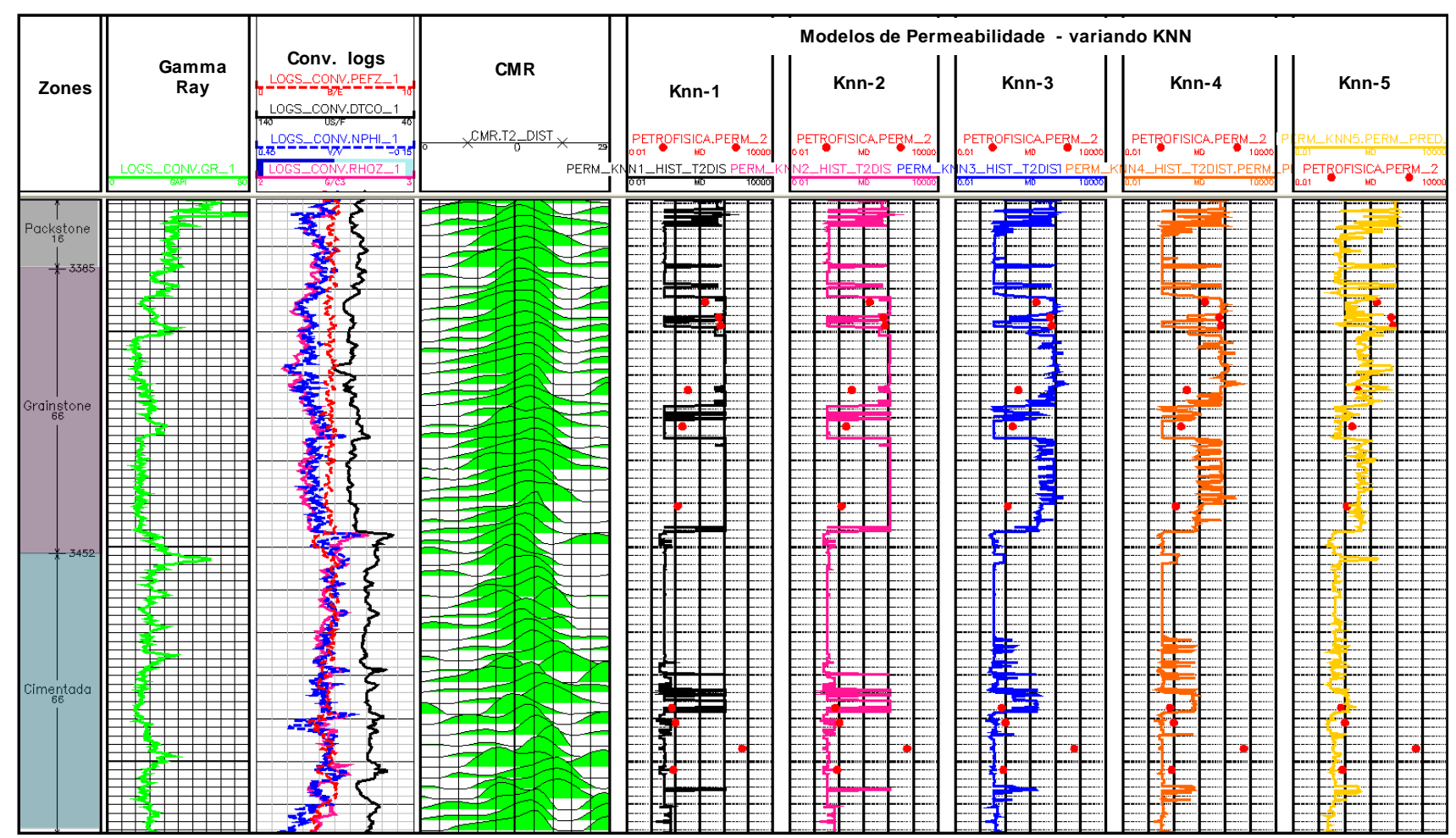

Figura 8. de permeabilidade variando o KNN (Kernel Nearest Neighbours) no poço B. 